etc., necessitated by the use of diets in the form of dry pellets and cubes (Bruce and Parkes ${ }^{1,2}$ ), made urgent the elaboration of a more efficient water container.

We have therefore evolved a drinking spout which can be attached to the cap of almost any standard screw-topped bottle. The spout is made from $\frac{3}{8}$-in. external diameter 18-gauge metal tubing. The distal end is rounded by spinning to form a vent about $\frac{1}{8}$-in. diameter. The proximal end is threaded for about half an inch, and a thin hexagon nut is screwed on up to the end of the thread. The end of the tube is then passed through a $\frac{3}{8}$-in. hole pressed in the bottle top and secured by a second nut inside the cap. Both nuts are coated with 'Bostic' to ensure an air-tight joint. $A$ washer inside the cap ensures an air-tight joint with the bottle. Spouts of this type can be made of any appropriate length and shape.

Two types are now in routine use at the National Institute for Medical Research. The first is $6 \mathrm{in}$. in length with a slight bend, and is attached to the aluminium cap of the standard blood transfusion bottle for use with large cages (for example, rabbit cages), or to the cap of the standard 10-oz. flat bottle for use with smaller cages. The bend in the spout enables it to be used in the horizontal position on top of the cage or in the vertical position on the outside of the cage, to which it is attached by means of a wire holder. Where the cap of the bottle is large, as with the blood transfusion type, it should be strengthened with an 18-gauge disk inside each nut. The second type of spout is short (2 in.) and straight, and is used on the plastic cap of a small squat bottle (ink bottle type) for rat isolation cages or mouse boxes. The spouts can be moved from one type of bottle cap to another as required. The present spouts are made of 'Dural' tubing, and the price is very moderate. The short spout and squat bottle together cost about 25 per cent of the present price of the fragile glass bulb which they replace completely. It is likely, however, that the 'Dural' tubing will prove too soft for use with guinea pigs, and that brass spouts will be required for this species.

We are indebted to Dr. B. Wheeler Robinson for suggestions on design. The equipment described was made by Herbert $W$. Baker (Engineering), Ltd., St. John's Works, 2a Kilburn Lane, London, W.10.

$$
\text { D. J. SHORT }
$$

A. S. PARKes

National Institute for Medical Research,

London, N.W.3.

Oct. 30 .

'Bruce, H. M., and Parkes, A. S., J. Hygiene, 44, 501 (1946).

'Bruce, H. M., and Parkes, A. S., J. Hygiene, 45, 70 (1947).

\section{Science in the East African Groundnut Scheme}

Sir John Russell, stressing, in his address to the lo Play Society at Oxford on December 31, the importance of adequate preparatory experimental work before developments such as the groundnut scheme are undertaken, is reported (The Times, January 1) as having said that the scientific and technical staff of the scheme "never really had a chance to do their work properly" and that they were "not allowed the necessary time" to make small-scale experiments to discover the snags and difficulties of the scheme. He is also said to have stated that "those who had been experimenting" in the scheme "were very disappointed with the results".

These reports may give rise to impressions other than those intended by Sir John Russell, and on two points in particular clarification is necessary.

First, it is true that the scheme was started in advance of any detailed experimental work in the areas concerned. This course was dictated by the urgency of the world food position, since it was believed on the technical information and experience available in 1946 that the general conditions were adequate for the economic production of groundnuts and other crops in the areas designated.

This proposition has from the first been under test. I was a member of the advance party which reached Tanganyika early in February 1947, and the scheme's Scientific Department, which now includes seventeen European scientific and technical workers, together with a growing corps of African scientific assistants, has carried out a considerable programme of soil and vegetation surveys, followed by laboratory and field studies of soil fertility, alternate cropping and rotations, and plant protection problems.

Three experiment stations, each of several hundred acres, have been developed in the central, western and southern provinces of Tanganyika. The headquarters, at Kongwa, include a tented laboratory, a library and a statistical section. These studies have done much to confirm the conclusions of the Wakefield mission on agricultural questions, at least so far as Tanganyika is concerned.

It is true, of course, that the scientific and tech. nical effort will need considerable expansion if the problems of establishing, in a matter of ten years or so, a system of large-scale mechanized agriculture over millions of acres of new land in East Africa are to be dealt with adequately. The need is particularly acute in respect of laboratories. We meet, inevitably, many difficulties of supplies and com. munications; but we have been well supported by the management of the scheme, whether under the United Africa Company or the Overseas Food Cor. poration.

Secondly, the many delays and difficulties of the last two years, which are as disappointing to the technical staff as to all other workers on the project, are not in the main due to lack of technical information such as agricultural research can be expected to provide, nor, as has been alleged by some, to disastrous failures on the agricultural side. They arise in great part from engineering problems of clearing, construction, communications and general development, which have proved to be greater than was foreseen, and could not have been eliminated by small-scale research, since they are essentially prob. lems of large-scale operation and organisation in new country. These very delays give us on the research side the more time to press forward our investigations.

This is not to say that the agricultural problems are negligible. They are many and important; some promise to give much trouble. Nevertheless the scientific and technical staff share with Sir John Russell, under whom many of us formerly served, the belief that we shall find the solutions.

\section{A. H. Bunting}

Overseas Food Corporation, (Chief Scientific Officer) 